\title{
Semimicroscopic Dispersive Analysis of Nuclear-Nuclear Collisions on the Basis of Folding Potential
}

\author{
Abdolmajid Izadpanah', Marzieh Yousefi² \\ ${ }^{1}$ Department of Physics, Golestan University, Gorgan, Iran \\ ${ }^{2}$ Department of Physics, School of Fariman Payame Nor, Fariman, Iran \\ Email: amjizad@gmail.com
}

Received 16 February 2014; revised 12 March 2014; accepted 7 April 2014

Copyright (C) 2014 by authors and Scientific Research Publishing Inc.

This work is licensed under the Creative Commons Attribution International License (CC BY). http://creativecommons.org/licenses/by/4.0/

c) (i) Open Access

\begin{abstract}
A semimicroscopic analysis of a set of experimental data of elastic $\alpha+{ }^{12} C$ scattering was performed at several laboratory energies. The Woods-Saxon parameters were adjusted to obtain the best $\chi^{2}$ fit to the scattering data. The energy systematics of the positions of Airy minima was constructed, and it was shown that their positions depend linearly on the inverse center of mass energy. The parameters of the model potential have been determined unambiguously. It has been shown that the energy dependence of the volume integrals satisfies the dispersion relation and agrees well with the results obtained within a phenomenological analysis. Also, it has been shown that the found positions of the Airy minima satisfy the rule of the quadratical dependence of the position of the Airy minima on the reduced mass of the colliding nuclei.
\end{abstract}

\section{Keywords}

Nuclear Dispersion Anomaly, Optical Model Potential, Nuclear Rainbow, Airy Minima

\section{Introduction}

The essence of the potential approach, that has been applied to describe elastic scattering and direct reaction in nucleus-nucleus collisions at energies in the range extending up to $100 \mathrm{MeV}$ per nucleon, is that the system of two interacting nuclei at the given energy in the elastic channel can be described by a model-dependent wave function that is found by solving the single-particle Schrödinger equation with an effective potential [1]. Effective potential is nonlocal, complex-valued and energy-dependent and can be represented as the sum of two components: static component or "mean-field potential” that represents the interaction of nuclei in their ground 
states and is energy-independent and dynamical component or "dynamical polarization potential" (DPP) that carries information about all possible inelastic channels of the interaction between colliding nuclei.

The new and important point in this work is the calculation of the dispersion relation between the real and imaginary parts of the dynamical polarization potential. This relation is a result of the causality principle: a scattered wave cannot be emitted before the interaction has occurred [2].

The model wave function may be written in terms of the complete set of internal wave functions of the projectile $(a)$ and target $(A)$ nuclei as [1]

$$
\psi_{\text {model }}=A\left[u(r) \psi_{a}\left(x_{a}\right) \psi_{A}\left(x_{A}\right)\right],
$$

where $\psi_{i}\left(x_{i}\right)$ is the internal wave functions of colliding nuclei in their ground states. The problem is reduced to the single-particle Schrödinger equation for scattering wave function $u(r)$ that describes the motion of the particle with reduced mass $\mu$ in effective complex potential:

$$
\left[-\frac{\hbar^{2}}{2 \mu} \nabla^{2}+u\right] u^{(+)}(r)=E u^{(+)}(r) .
$$

This equation is the basis of the optical model formulation that its essence is the construction of the effective potential model can be written

$$
u(r)=-V f\left(x_{V}\right)-i W f\left(x_{W}\right)+i 4 W_{D} \frac{\mathrm{d} f\left(x_{D}\right)}{\mathrm{d} x_{D}}+V_{C}(r),
$$

where $f(x)=\left(\mathrm{e}^{x}+1\right)^{-1}, \quad x_{i}=\frac{r-R_{i}}{a_{i}}(i=V, W, D)$, is the Woods-Saxon form factor. Here $V_{\mathrm{c}}(r)$ is Coulomb potential. Strength parameters $(V, W)$ "depths of potential" and geometric parameters (radii and diffuseness) are obtaining using the analysis of the elastic scattering experimental angular distributions. The parameters are adjusted to obtain the best $\chi^{2}$ fit to the scattering data.

One of the approaches to construction of effective potential is microscopic approach: attempt to understand interaction of two nuclei in terms of the motions of individual nucleons and their interactions. Static component, that is the matrix element of the real effective nucleon-nucleon interaction, is the ground states wave functions of colliding nuclei

$$
\left(V_{p p}\right) \equiv V_{F}=\left\langle\psi_{a_{0}} \psi_{A_{0}}|V A| \psi_{a_{0}} \psi_{A_{0}}\right\rangle,
$$

In this relation, already, dual nucleon-nucleon interaction, $V=\sum_{p=1}^{a} \sum_{t=1}^{A} v_{p t}$, is describing ( $t$ and $p$ are used for target and projectile nuclei, respectively) in generally this interaction is shown as

$$
v_{p t}=\sum_{T=0,1} \sum_{S} g_{S T}^{C}(S) \sigma_{p}^{S},
$$

where $S=r_{p}-r_{t}+r$ is vector-radius according to nucleon $p$ in the projectile $a$, and nucleon $t$ in the target $A$.

The radial functions of the central component, $g_{S T}^{C}(S)$, spin-orbital component, $g_{T}^{S o}(S)$, tensor component, $g_{T}^{\text {Ten }}(S)$, and Coulomb component, $g_{C}(S)$ (for protons) are the functions of the distance between given pair nucleons. Here, $v_{T}$ is the isoscalar $(T=0)$ and isovector $(T=1)$ components of the effective nucleonnucleon interaction. The M3Y model of effective nucleon-nucleon interactions in one of two versions, that which involves the input Reid-Elliott potential [3] and that which involves the input Paris potential [4], is used to calculate the mean-field potential.

The radial part of the various components of interaction is shown by the sum of the Yukawa's potentials. But this model of interaction is averaged over the energy and density and is not dependent on them explicitly. Obviously, it is very difficult to abandon the energy and density dependence. The density dependence is included in effective nucleon-nucleon interactions (central direct and exchange components in this case) in the form of a density-dependent factor multiplied by the M3Y model interaction (indices being suppressed):

$$
g(S, p)=f(p) g(S) .
$$

In the present study, we use the parametrization 


$$
f(p)=C\left[1+\alpha \mathrm{e}^{-\beta \rho}-\gamma \rho\right]
$$

in the CDM3Y6 version, where the parameters involved were fitted to the properties of cold nuclear matter [5], but in contrast to [5], here is not introduced additional energy dependence. The single-nucleon knockout exchange (SNKE) between the two ions will be the dominant contribution, especially for light ions, even in the multichannel case. In the approximation of single-nucleon knockout exchange, the mean-field potential can be expressed in terms of folding integrals for the direct and exchange components. Omitting spin variables and spin components, it can written

$$
V_{F}=V^{D}+V^{E}
$$

where $V^{D}$ is the direct component

$$
\begin{aligned}
V^{D}(r) & =\sum_{T=0,1} V_{T}^{D}(r), \\
V_{T}^{D}(r) & =\int \mathrm{d} r_{1} \mathrm{~d} r_{2} g_{T}^{C, D}(s) \rho_{T}^{a}\left(r_{1}\right) \rho_{T}^{A}\left(r_{2}\right)
\end{aligned}
$$

and $s=r_{1}-r_{2}+r, \rho_{T}^{i}$ stands for the isoscalar $(T=0)$ or the isovector $(T=1)$ nuclear-density component $(i=a, A)$ which are related in a standard way to the proton- and neutron-density distributions in each nucleus. The localized exchange component is given by

$$
\begin{aligned}
V^{E}(r) & =\sum_{T=0,1} V_{T}^{E}(r) \\
V_{T}^{E}(r) & =\int \mathrm{d} r_{1} \mathrm{~d} r_{2} g_{T}^{C, E}(s) \rho_{T}^{a}\left(r_{1}, r_{1}+s\right) \rho_{T}^{A}\left(r_{2}, r_{2}-s\right) \exp \left(\frac{i K(r) s}{\mu}\right)
\end{aligned}
$$

Here $\rho_{T}^{i}\left(r_{1}, r_{2}\right)$ are the corresponding components of the nuclear density matrices. The local momentum $K(r)$ of relative motion is written as

$$
K^{2}(r)=\frac{2 \mu}{\hbar^{2}}\left(E-V(r)-V_{C}(r)\right)
$$

where $V_{C}(r)$ is the Coulomb potential.

The density matrix, usually, is made with the aid of the modified Slater approximation (indices are suppressed)

$$
\rho(r, r+s)=\rho\left(r+\frac{s}{2}\right) j_{1}\left(k_{e f f}\left(r+\frac{s}{2}\right) s\right)
$$

where $j_{1}(\chi)=3(\sin (\chi)-\chi \cos (\chi)) / \chi^{3}$ is the exchange correlation function. The effective-momentum components are determined by an obtained expression from the generalized Thomas-Fermi approximation for the kinetic-energy density

$$
\left(k_{\text {eff }}^{i}\right)^{2}=\left(\frac{3}{2} \pi^{2} \rho_{0}^{i}\right)^{2 / 3}+\frac{5}{3}\left(\frac{\nabla \rho_{0}^{i}}{6 \rho_{0}^{i}}\right)^{2}+\frac{5}{36} \frac{\nabla^{2} \rho_{0}^{i}}{\rho_{0}^{i}} .
$$

It was shown that, in the absence of the density dependence of nucleon-nucleon forces, the result of the calculation with the aid of (12) and (13) is virtually coincident with the result of the exact calculation. The use of the different version of the approximation in (13) leading to a modest distinction. Upon the inclusion of the density dependence, however, the deviation in both versions from the result of the "exact" calculation becomes sizable (smaller for the first, but greater for the second version) especially at low energies. All versions of the calculation of nuclear density matrices are rather cumbersome. In order to simplify the calculations, an approximate representation of the density matrix is used in the form [6]

$$
\rho_{A}\left(r+\frac{s}{2}, r-\frac{s}{2}\right)=\rho_{A}(r) \exp \left[-\frac{s^{2}}{4 b_{A}}\right]
$$

where an empirical nuclear density for $\rho_{A}(r)$ is used and where 


$$
b_{A}=b_{0} A^{1 / 3} .
$$

For $b_{0}=0.92 \mathrm{fm}^{2}$ the approximation in (14) for the case of the alpha particle is coincident with the density matrix calculated within the harmonic oscillator model. Therefore, this representation was called a pseudooscillator approximation. Clearly, the microscopic calculation of the dynamical component of the effective potential is difficult problem.

Other approach, that is widely used to construct effective potential, is called dispersion semimicroscopic approach. A semimicroscopic approach combines a microscopic calculation of the mean-field potential and a phenomenological construction of the dynamical polarization potential.

In this model, the dynamical polarization potential is constructed on the basis of physically justified combinations of the volume and surface forms whose geometric parameters are assumed to be independent of energy.

Furthermore, in this model the imaginary part of the central component DPP is represented as the sum of the volume, $W(r, E)$, and the surface, $W_{D}(r, E)$, part and they are shown by the Woods-Saxon form and its derivative. In this model, the optical model potential (Equation (2)) is represented in the form

$$
U=V_{F}+V_{P}+i W+V_{C},
$$

where the first term is the mean-field potential being calculated, the second term is the real part of the phenomenological dynamical polarization potential (dispersive correction), and the third term is its imaginary (absorptive) part. The absorption includes the volume $(W)$ and surface $\left(W_{D}\right)$ components. Here, they are represented by

$$
i W=-i W(E) f\left(x_{W}\right)+i 4 W_{D}(E) \frac{\mathrm{d} f\left(x_{D}\right)}{\mathrm{d} x_{D}},
$$

where $f(x)=\left(\mathrm{e}^{x}+1\right)^{-1}, \quad x_{W}(D)=\frac{r-R_{W}(D)}{a_{W}(D)}$ with $R_{W}(D)=r_{W}(D) A^{1 / 3}$ in the Woods-Saxon form factor, and the dispersive correction is given by the expression

$$
V_{P}(r, E)=\alpha(E) W(E) f\left(x_{W}\right)+\beta(E) 4 W_{D}(E) \frac{\mathrm{d} f\left(x_{D}\right)}{\mathrm{d} x_{D}},
$$

( $\alpha$ and $\beta$ are free parameters) which follows from the dispersion relation if one assumes the energy independence of geometric parameters (radius and diffuseness of the Woods-Saxon form).

In this study, the Coulomb component $V_{C}$ is simulated by the interaction potential of a pointlike charge with a uniformly charged sphere of radius $R_{C}$ that is given by [1]

$$
R_{C} \approx\left[\frac{5}{3}\left\langle r^{2}\right\rangle_{c h a}\right]^{1 / 2}+\left[\frac{5}{3}\left\langle r^{2}\right\rangle_{c h A}\right]^{1 / 2}
$$

Keeping simplicity and convenience of application, such model effectively has smaller number of parameters and allows reducing ambiguity of the analysis.

The dispersive analysis of the volume integrals was performed here on the basis of the difference dispersion relation [7]

$$
J_{P}(E)-J_{P}\left(E_{S}\right)=\left(E-E_{S}\right) \frac{P}{\pi} \int \frac{J_{W}\left(E^{\prime}\right)}{\left(E^{\prime}-E_{S}\right)\left(E^{\prime}-E\right)} \mathrm{d} E^{\prime},
$$

where the dependence $J_{W}(E)$ was approximated on the basis of the linear schematic model [2]. Here, the volume integral of the real part of the potential is the sum of two terms,

$$
J_{V}(E)=J_{F}(E)+J_{P}(E),
$$

corresponding to the mean-field potential and the dispersion correction.

\section{Semimicroscopic Dispersive Analysis of Elastic $\alpha+{ }^{12} C$ Scattering}

In order to analysis of experimental data, in this work, we used angular distributions for elastic $\alpha+{ }^{12} C$ scatter- 
ing that are available from the NNDC (EXFOR/CSISRS) database (http://www.nndc.bnl.gov/exfor). An opticalmodel analysis was performed in fixing the geometric parameters $r_{W}=1.03 \mathrm{fm}, a_{W}=0.61 \mathrm{fm}, r_{D}=0.258 \mathrm{fm}$, $a_{D}=0.47 \mathrm{fm}$. These parameters were obtained in an individual analysis involving a search for various combinations of geometric parameters, over a reasonable interval of their values.

The values found for the remaining parameters and values of $\frac{\chi^{2}}{N_{\sigma}}\left(N_{\sigma}\right.$ is the number of experimental points in a given angular distribution) are given in Table 1 for each data set in energy under consideration. As a rule, the analysis was performed by using a constant experimental error of 10 percent.

Figure 1 shows the ratios of the experimental and calculated differential cross sections for elastic $\alpha+{ }^{12} C$ scattering at various laboratories energies to the respective Rutherford cross sections. Nuclear rainbow pattern clearly indicated in all energies and so it is possible to construct energy systematics of the Airy minima positions.

In this work, three tests were used to avoid ambiguities in determining the potential: due to of rainbow structure, it is possible to construct energy systematics of the Airy minima positions. This systematics showed and confirmed the inverse-energy law and made it possible to select potential (see Figure 2). In Figure 2, the circles and triangles are indicated experimental Airy minima, while the stars are our calculated Airy minima. Lines 1 and 2 are corresponding to the positions of the first and second Airy minima, respectively. The results of the dispersive analysis using phenomenological approach (squares) and also semimicroscopic approach (circles) are displayed in Figure 3. This is second test. Both two approaches are shown some behaviors.

The curve introduced by Goncharov and Izadpanah [8], for depending of Airy minima to reduced mass of col-

Table 1. Found parameters and integral volumes of potential for $\alpha+{ }^{12} \mathrm{C}$ system.

\begin{tabular}{|c|c|c|c|c|c|c|c|c|}
\hline$E_{l a b}(\mathrm{MeV})$ & $-\alpha$ & $-\beta$ & $V(\mathrm{MeV})$ & $W(\mathrm{MeV})$ & $\begin{array}{c}-J_{V} \\
\left(\mathrm{MeV} \cdot \mathrm{fm}^{3}\right)\end{array}$ & $\begin{array}{c}-J_{W} \\
\left(\mathrm{MeV} \cdot \mathrm{fm}^{3}\right)\end{array}$ & $\sigma(\mathrm{mb})$ & $\chi^{2} / N_{\sigma}$ \\
\hline 104 & 0.186 & 0.236 & 15.28 & 16.94 & 339.5 & 118.4 & 825 & 8.5 \\
\hline 120 & 0.120 & 0.20 & 16.76 & 7.55 & 340.4 & 120.8 & 821 & 3.2 \\
\hline 139 & 0.559 & 0.466 & 14.89 & 7.49 & 287.7 & 108 & 757.9 & 10.4 \\
\hline 145 & 0.158 & 0.230 & 16.79 & 7.9 & 325.5 & 121.3 & 788.7 & 4.1 \\
\hline 166 & 0.163 & 0.391 & 15.72 & 14.8 & 314.5 & 119.7 & 753 & 6.5 \\
\hline 172.5 & 0.129 & 0.142 & 17.49 & 4.17 & 318.4 & 123 & 769.3 & 5.1 \\
\hline 240 & 0.485 & 0.357 & 13.78 & 6.21 & 262.9 & 99.4 & 650.5 & 3.4 \\
\hline
\end{tabular}

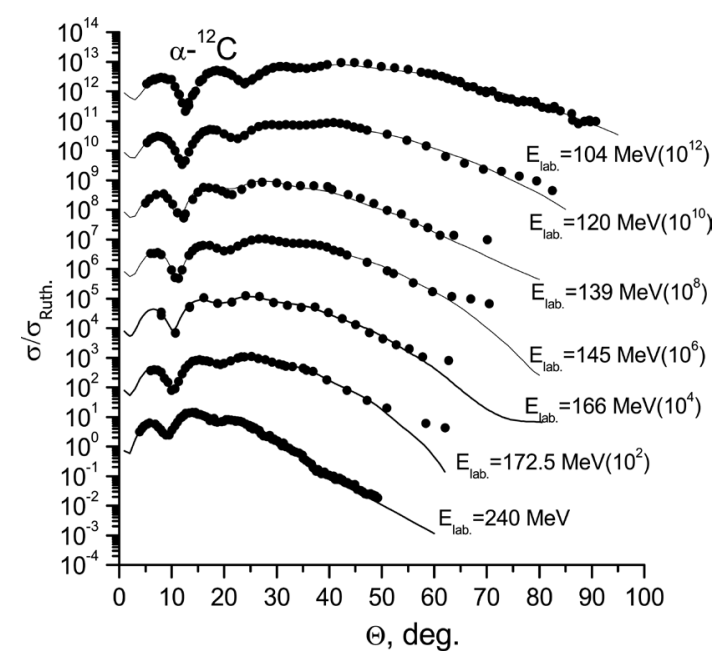

Figure 1. Ratios of the differential cross sections for elastic scattering $\alpha+{ }^{12} C$ system. 


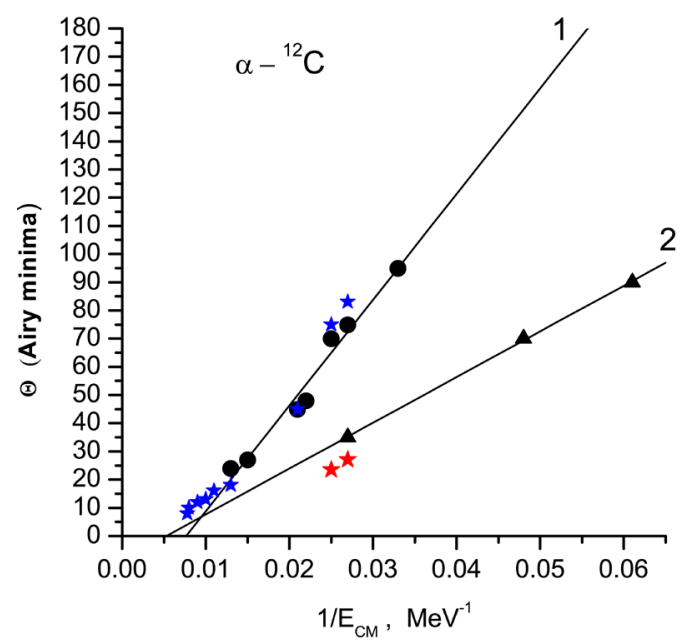

Figure 2. Positions of the Airy minima for $\alpha+{ }^{12} \mathrm{C}$ system.

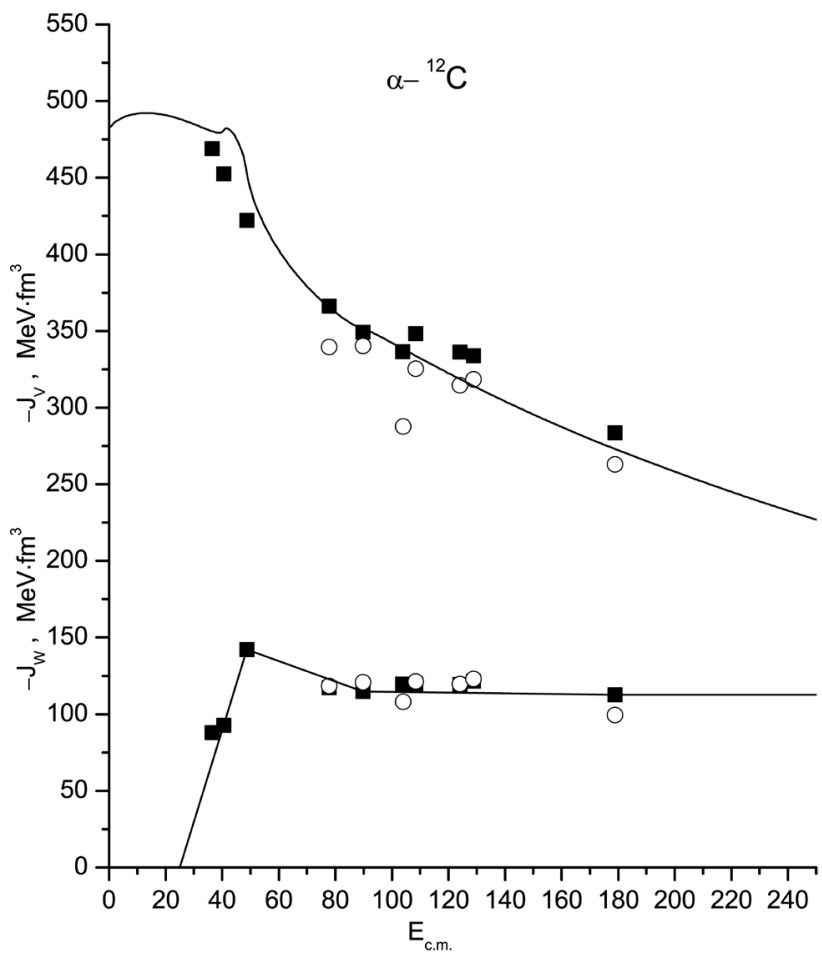

Figure 3. Dispersion analysis of volume integrals of dispersive potentials for the $\alpha+{ }^{12} C$ system.

liding nuclei, third test, has been shown in Figure 4. In this figure, the stars showed in the energies (center of mass) of 80 and $120 \mathrm{MeV}$ in first Airy minima are the results that, clearly, are satisfactory.

The farside components calculated by us have been shown in Figure 5. As we expected, the Airy minima shifts toward small degrees with increasing energy of projectile. The radial dependence of the calculated total real $(V)$ and the total imaginary $(W)$ parts of the potentials at various laboratory energies have been shown in Figure 6.

\section{Conclusions}

A semimicroscopic analysis of a set of experimental data of elastic $\alpha+{ }^{12} C$ scattering was performed at several 


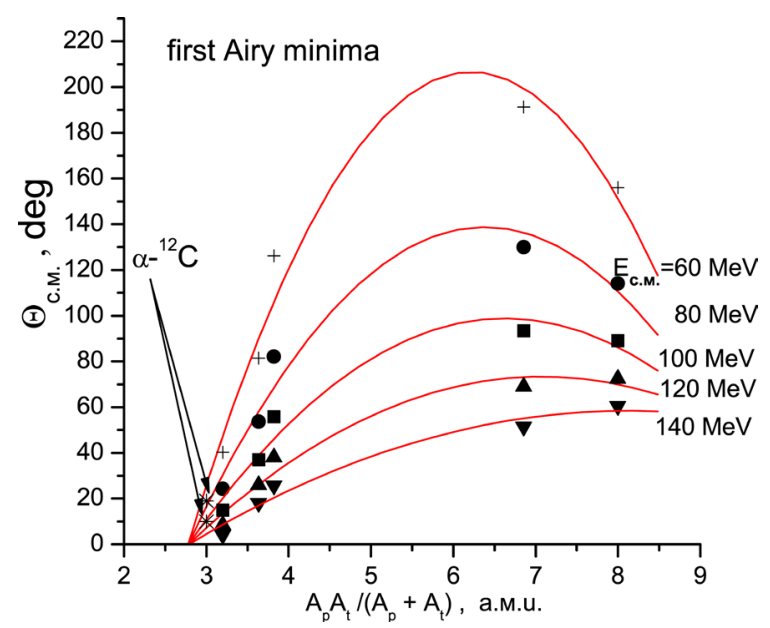

Figure 4. Estimated positions and approximating secondorder polynomials curves of first Airy minima versus the reduced mass.

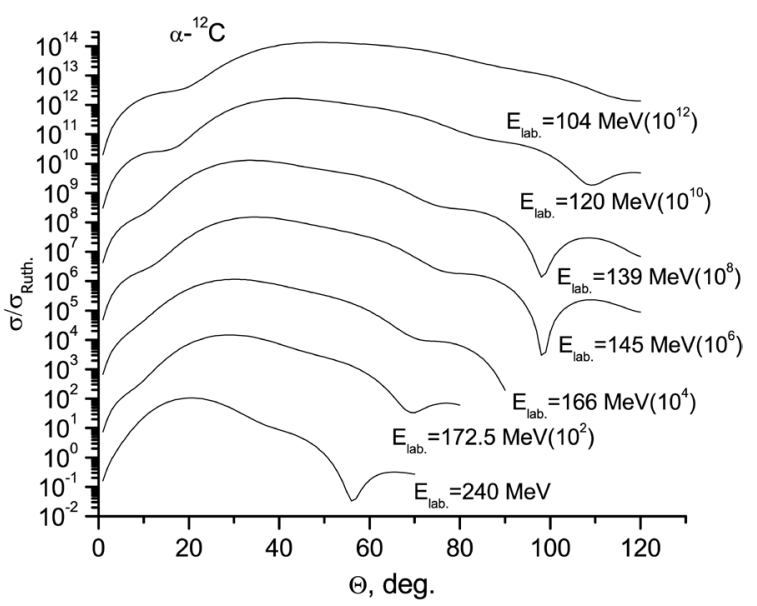

Figure 5. Calculated farsaid components for $\alpha+{ }^{12} C$ system.

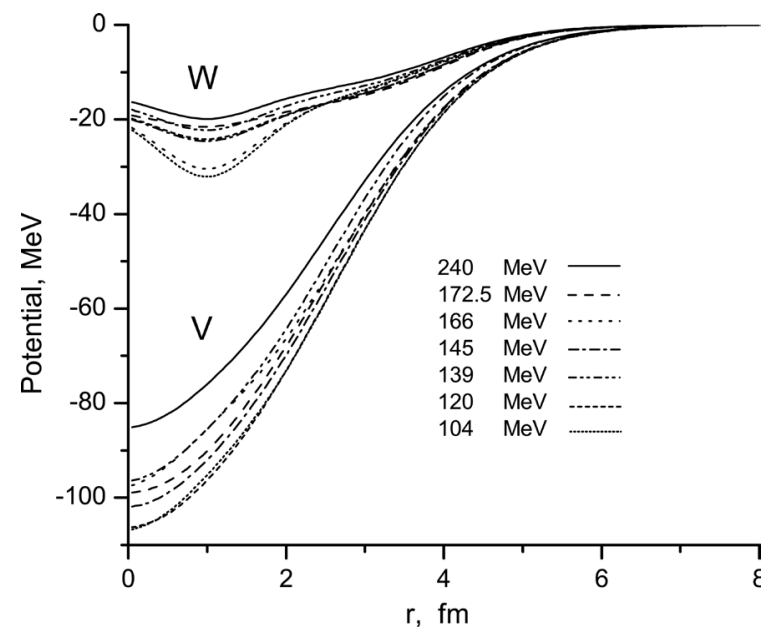

Figure 6. Found radial dependence of the total real and imaginary part of potential for $\alpha+{ }^{12} C$ system at various laboratory energies. 
laboratory energies. In this way, the microscopic calculation of static component of potential combines with the phenomenological calculation of the dynamical component of potential. In this model, the dynamical polarization potential is constructed on the basis of physically justified combinations of the volume and surface forms whose geometric parameters are assumed to be independent of energy.

The energy systematics of the positions of Airy minima was constructed, and it was shown that their positions depend linearly on the inverse center of mass energy. The energy dependence of the volume integrals was performed and has been shown that the dispersion relation is satisfied and agrees well with the results obtained within a phenomenological analysis. Using systematics of the positions of Airy minima with respect to reduced mass of colliding nuclei introduced by Goncharov and Izadpanah, has been shown that the found positions of the Airy minima satisfy the rule of the quadratical dependence of the position of the Airy minima on the reduced mass of colliding nuclei.

Using these three systematics, the parameters of the model potential at various energies have been determined unambiguously.

The obtained imaginary components are shown the meaningness of aberrations from its Woods-Saxon forms obtained using the phenomenological approach. This aberration occurs in the distances about $1 \mathrm{fm}$ and one can be due to ignore of the dependence energy in this study.

\section{Acknowledgments}

We are grateful to C.A. Goncharov for enlightening comments and discussions.

\section{References}

[1] Satchler, G.R. (1983) Direct Nuclear Reactions. Clarendon, Oxford, 392.

[2] Mahaux, C., Ngo, H. and Satchler, G.R. (1986) Nuclear Physics A, 449, 354. http://dx.doi.org/10.1016/0375-9474(86)90009-6

[3] Bertsch, G., Borysowics, J., McManus, H. and Love, W.G. (1977) Nuclear Physics A, 284, 399. http://dx.doi.org/10.1016/0375-9474(77)90392-X

[4] Anantaraman, N., Toki, H. and Bertsch, G. (1983) Nuclear Physics A, 398, 269. http://dx.doi.org/10.1016/0375-9474(83)90487-6

[5] Khoa, D.T., Satchler, G.R. and von Oertzen, W. (1997) Physical Review C, 56, 954. http://dx.doi.org/10.1103/PhysRevC.56.954

[6] Goncharov, S.A. and Izadpanah, A. (2007) Physics of Atomic Nuclei, 70, 18. http://dx.doi.org/10.1134/S1063778807010036

[7] Ogloblin, A.A., Goncharov, S.A., Glukhov, Yu.A., et al. (2003) Physics of Atomic Nuclei, 66, 1478. http://dx.doi.org/10.1134/1.1601753

[8] Goncharov, S.A. and Izadpanah, A. (2007) Physics of Atomic Nuclei, 70, 1491. http://dx.doi.org/10.1134/S1063778807090037 
Scientific Research Publishing (SCIRP) is one of the largest Open Access journal publishers. It is currently publishing more than 200 open access, online, peer-reviewed journals covering a wide range of academic disciplines. SCIRP serves the worldwide academic communities and contributes to the progress and application of science with its publication.

Other selected journals from SCIRP are listed as below. Submit your manuscript to us via either submit@scirp.org or Online Submission Portal.
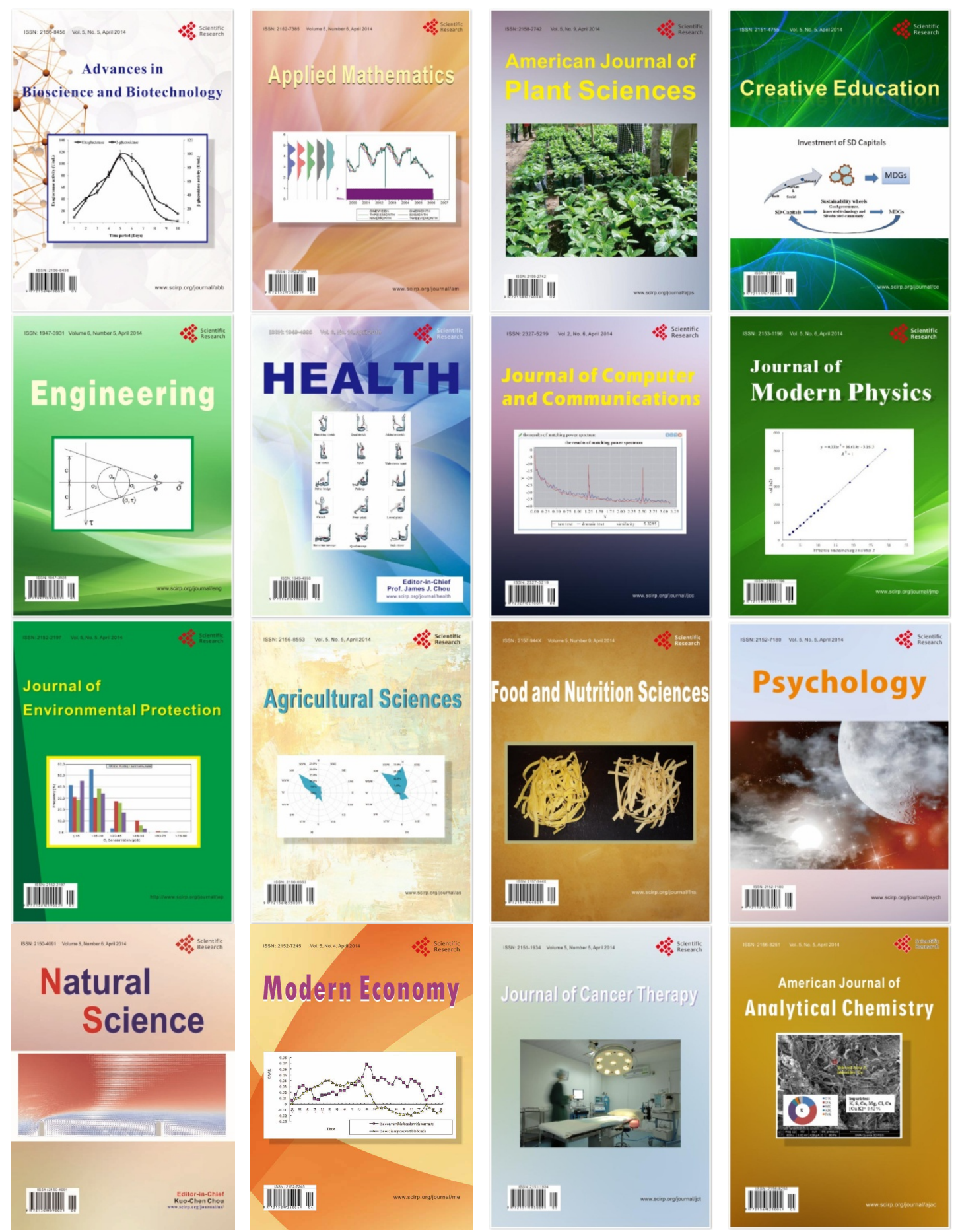